\title{
a-Glucosidase inhibitory activities, functional properties, and safety of green tea polysaccharides as a potential source of dietary supplement
}

\author{
Ayse Karadag ${ }^{\mathrm{a}}$, Kubra Dogan ${ }^{\mathrm{a}}$, Ebru Pelvan ${ }^{\mathrm{b}}$, Arzu Tas $^{\mathrm{c}}$, Ozlem Atli Eklioglu ${ }^{\mathrm{d}}$, \\ Soner $\mathrm{Aksu}^{\mathrm{c}}$ and Cesarettin Alasalvar ${ }^{\mathrm{b}^{*}}$
}

\begin{abstract}
aDepartment of Food Engineering, Y1ldız Technical University, 34210, Esenler-İstanbul, Turkey
bTÜBITTAK Marmara Research Center, Food Institute, P.O Box 21, 41470 Gebze-Kocaeli, Turkey

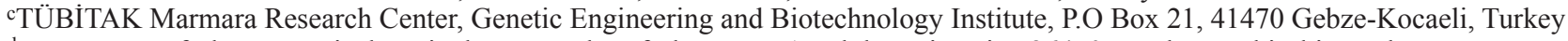

dDepartment of Pharmaceutical Toxicology, Faculty of Pharmacy, Anadolu University, 26470 Tepebaş1-Eskişehir, Turkey

*Corresponding author: Cesarettin Alasalvar, TÜBİTAK Marmara Research Center, Food Institute, P.O Box 21, 41470 Gebze-Kocaeli, Turkey. Tel: +90 262677 3200; E-mail: cesarettin.alasalvar@tubitak.gov.tr
\end{abstract}

DOI: $10.31665 /$ JFB. 2018.3155

Received: July 11, 2018; Revised received \& accepted: September 04, 2018

Citation: Karadag, A., Dogan, K., Pelvan, E., Tas, A., Eklioglu, O.A, Aksu, S., and Alasalvar, C. (2018). $\alpha$-Glucosidase inhibitory activities, functional properties, and safety of green tea polysaccharides as a potential source of dietary supplement. J. Food Bioact. 3: $124-132$.

\begin{abstract}
Procuring valuable bioactive compounds from low quality green tea is of great interest. Green tea polysaccharides (GTPS), obtained from low quality tea leaves, were examined for their yield, monosaccharide composition, total phenolic content, antioxidant potential, and structures as well as molecular weight distribution and in vitro anti-diabetic activities. GTPS were also evaluated for their toxicity by performing cytotoxicity and genotoxicity. Additionally, $50 \%$ lethal dose $\left(\mathrm{LD}_{50}\right)$ for GTPS was determined using an acute toxicity test to assess the safe use of it as a dietary supplement. Monosaccharides of GTPS were mainly composed of xylose, glucose, ribose, galactose, arabinose, and glucuronic acid. GTPS exhibited $91.86 \% \alpha$-glucosidase inhibition at $0.5 \mathrm{mg} / \mathrm{mL}$ concentration. The $\alpha$-glucosidase inhibitory activity of GTPS was higher than that of pharmacological glucosidase inhibitor, acarbose, which exhibited only $47.86 \%$ inhibition at $2.5 \mathrm{mg} / \mathrm{mL}$ concentration. Neither cytotoxic nor no mutagenic effects were found for GTPS. The acute toxicity also showed that $\mathrm{LD}_{50}$ of GTPS was greater than $5,000 \mathrm{mg} / \mathrm{kg}$. The present work suggests that GTPS can be considered as an anti-diabetic dietary supplement without posing any potential health risk.
\end{abstract}

Keywords: Green tea polysaccharide (GTPS); Anti-diabetic; $\alpha$-Glucosidase inhibition; Toxicity tests; $\mathrm{LD}_{50}$ value; Dietary supplement.

\section{Introduction}

Diabetes mellitus (DM) has become one of the biggest public health challenges for the $21^{\text {st }}$ century around the world and type II diabetes is the most common form which accounts for $90-95 \%$ of all diabetes cases. Exploration of novel safe and effective bioactive compounds with anti-diabetic activity has become an important research focus due to the serious adverse effects associated with the oral synthetic hypoglycemic agents (Shori, 2015; Wang and

\section{Zhu, 2016).}

Tea is one of the most popular beverages around the globe. The majority of research has recently been concentrated on the understanding of the molecular characterization and bioactivities of complex tea polyphenols. Over the last decade, tea polysaccharides have also attracted scientific interest due to their antidiabetic effects (Chen et al., 2016; Nie and Xie, 2011; Xiao and Jiang, 2015).

Turkey is the fifth largest producer of tea, with a production of 221,600 metric tonnes (Alasalvar et al., 2013). The products 
present in Turkish market are loose tea, tea bag, and flavoured tea. Therefore, an alternative use of tea as raw material for dietary supplement is of great interest for both producers and consumers. Although an increasing number of studies indicate the possible anti-diabetic activity of tea polysaccharides, there is limited information available in the literature about their potential toxicity. The aims of this study were to examine the physico-chemical characteristics, functional properties, and in vitro anti-diabetic activities of green tea polysaccharides (GTPS) and to assess their toxicity using various assays (including an acute toxicity) to facilitate their potential use as a source of dietary supplement.

\section{Materials and methods}

\subsection{Samples and reagents}

Green tea samples (Antik Yeşil I) were procured from ÇAYKUR (State-Owned Tea Enterprise, Rize, Turkey). All chemical reagents were obtained from Sigma-Aldrich Co. Ltd. (Dorset, UK), unless otherwise stated.

\subsection{Extraction of GTPS and determination of yield}

Green tea was mixed with $80 \%$ ethanol $(1: 10 ; \mathrm{w} / \mathrm{v})$ and allowed to stand overnight to remove most of the polyphenols, monosaccharides, and low-molecular-weight impurities. After filtration, tea leaves were air-dried and submitted to hot-water extraction with a extraction time of $120 \mathrm{~min}$, at a temperature of $80^{\circ} \mathrm{C}$, and ratio of water to tea leaves of 1:20 (m/v). After extraction, solutions were centrifuged at 5,870 $\mathrm{g}$ for $10 \mathrm{~min}$ and the supernatant was filtered through a filter paper (Whatman, grade 4, pore size 20-25 $\mu \mathrm{m}$; Fisher Scientific, Loughborough, UK). The filtrate was collected and concentrated to $1 / 4$ of the original volume with a rotary evaporator at $50{ }^{\circ} \mathrm{C}$ under vacuum. The filtrate was subsequently mixed with four volumes of ethanol and kept at $4{ }^{\circ} \mathrm{C}$ overnight. The precipitates were obtained by centrifugation at $15,000 \mathrm{~g}$ for $20 \mathrm{~min}$ at chill temperature $\left(4^{\circ} \mathrm{C}\right)$, and the pellet was washed with acetone and ethanol, solubilized in water, applied to reagent (butanol/ chloroform, v/v; 1:4), to remove proteins. The aqueous phase was dialyzed against distilled water for a day and lyophilized to obtain crude GTPS powder. The percentage yield of crude polysaccharide (Y) was calculated as follows:

$$
\mathrm{Y}(\%)=100 \times\left(\mathrm{W}_{\mathrm{p}} / \mathrm{W}_{\mathrm{i}}\right)
$$

where $\mathrm{W}_{\mathrm{p}}$ is the weight of the crude polysaccharide powder and $\mathrm{W}_{\mathrm{i}}$ is the weight of green tea.

\subsection{Characterization of GTPS}

\subsubsection{Determination of chemical composition}

Total carbohydrate was determined by the phenol-sulphuric acid method with D-glucose as a standard (DuBois et al., 1956; Xi et al., 2010). The soluble protein was determined by the method of Bradford (1976), using bovine serum albumin as a standard. The uronic acid content was determined by employing the $m$-hydroxylbiphenyl method described by Blumenkrantz and Asboe-Hansen
(1973) and using galacturonic acid as a standard.

\subsubsection{Determination of total phenolic content}

The content of total phenolics was determined according to the procedure described by Horszwald and Andlauer (2011), using the Folin-Ciocalteu phenol reagent. The content of total phenolics was calculated from a standard curve of gallic acid and expressed as grams of gallic acid equivalents (GAE) per $100 \mathrm{~g}$ of sample.

\subsubsection{Determination of antioxidant activities}

The two methods described by Cheng et al. (2013) were used to assess the 2,2'-azino-bis (3-ethylbenzothiazoline-6-sulphonic acid) (ABTS) radical scavenging activity and ferric reducing antioxidant potential (FRAP). Each sample was dissolved in water and the results were expressed as milligrams of trolox equivalents (TE) per 100 milligrams of sample for ABTS assay and $\mathrm{mg}$ of $\mathrm{FeSO}_{4} \cdot 7 \mathrm{H}_{2} \mathrm{O}$ equivalents per $100 \mathrm{mg}$ sample for FRAP assay.

\subsubsection{Determination of molecular weight}

The method described by Cai et al. (2013) was used to assess the molecular weight of the samples. Briefly, a high performance liquid chromatography (HPLC) system (LC-20AD pump, SPDM20A DAD detector, SIL-20A HT autosampler, CTO-2OAC column oven, DGU-20A5 degasser, and CMB-20A communications bus module; Shimadzu Corp., Kyoto, Japan) was used with a column (Ultrahydrogel Linear $7.8 \times 300$ mm; Waters Corp., Milford, MA, USA) and a guard column (Ultrahydrogel, $6 \times 40 \mathrm{~mm}$ ). The samples were dissolved $(10 \mathrm{mg} / \mathrm{mL})$ in the mobile phase solution and passed through a $0.45 \mu \mathrm{m}$ filter. The detailed conditions were as follows: mobile phase $\left(0.1 \mathrm{M} \mathrm{NaNO}_{3}\right)$, flow rate $(1 \mathrm{~mL} / \mathrm{min})$, column temperature $\left(45^{\circ} \mathrm{C}\right)$, and injection volume $(10 \mu \mathrm{L})$. Dextrans of different weights from Sigma were used as calibration standards (molecular weight: 1,000, 5,000, 12,000, 25,000, 50,000, 80,000, $150,000,270,000,410,000$, and 670,000 Da).

\subsubsection{Determination of monosaccharides}

The method described by Dai et al. (2010) was used to assess the monosaccharide composition of each sample. After hydrolysis of polysaccharides and derivatization of monosaccharides with 1-phenyl-3-methyl-5-pyrazolone (PMP), a HPLC system (LC20AD pump, SPDM20A DAD detector, SIL-20A HT autosampler, CTO-2OAC column oven, DGU-20A5 degasser, and CMB-20A communications bus module; Shimadzu Corp., Kyoto, Japan) was used with a Shiseido column (Capcell Pak C18, $250 \mathrm{~mm} \times 4.6 \mathrm{~mm}$, $5 \mu \mathrm{m}$ particles; Phenomenex Inc., Macclesfield, UK). The quantitation of monosaccharides was based on the calibration curves built for each of the standard.

\subsubsection{Fourier transform-infrared (FT-IR) spectrophotometric analysis}

The spectra of the polysaccharides were recorded with FT-IR spectrophotometer (Perkin Elmer Spectrum 400; Waltham, MA, USA). The finely powdered sample was pressed into the sample holder for FT-IR measurement in the frequency range of $4,000-650 \mathrm{~cm}^{-1}$. 
Table 1. Mutagenicity of compounds identified according to the evaluation criteria

\begin{aligned} & \hline Criteria Description \\ & \hline If baseline is $\leq 3 \\ & 2-3$ times greater than baseline weak mutagen \\ &$>3$ times greater than baseline mutagen \\ & If baseline is $>3 \\ & 1.5-2.5$ times greater than baseline weak mutagen \\ &$>2.5$ times greater than baseline mutagen \\ & \hline\end{aligned}

\subsubsection{Determination of $\alpha$-glucosidase activity}

The effects of extracts on $\alpha$-glucosidase activity were determined by modifying the method described by Wei et al. (2010), using $\alpha$-glucosidase from Saccharomyces cerevisiae (Sigma G5003; Sigma-Aldrich Co. Ltd., Dorset, UK). The substrate solution of 4-nitrophenyl $\alpha$-D-glucopyranoside (NPG) was prepared in 20 $\mathrm{mM}$ phosphate buffer with $6.7 \mathrm{mM}$ sodium chloride at $\mathrm{pH} 6.9$. A $75 \mu \mathrm{L}$ of $\alpha$-glucosidase $(1.0 \mathrm{U} / \mathrm{mL})$ was pre-incubated with $50 \mu \mathrm{L}$ of the different concentrations of the extracts for $5 \mathrm{~min}$. Then, a $75 \mu \mathrm{L}$ of $3.0 \mathrm{mM}$ of NPG as a substrate was added to start the reaction. The reaction mixture was incubated at $37{ }^{\circ} \mathrm{C}$ for $20 \mathrm{~min}$ and subsequently stopped by adding $2 \mathrm{~mL}$ of $0.1 \mathrm{M}$ $\mathrm{Na}_{2} \mathrm{CO}_{3}$. The $\alpha$-glucosidase activity was determined by measuring the yellow-coloured $p$-nitrophenol released from NPG at 405 $\mathrm{nm}$. One unit of $\alpha$-glucosidase activity was defined as the amount of enzyme required to produce one $\mu$ mole of $p$-nitrophenol from NPG $(3 \mathrm{mM})$ in sodium phosphate buffer $(20 \mathrm{mM})$ with $6.7 \mathrm{mM}$ $\mathrm{NaCl}, \mathrm{pH} 6.9$ at $37^{\circ} \mathrm{C}$. Percentage inhibition was calculated as follows:

$$
\text { Inhibition }(\%)=\left[\left(\mathrm{Abs}_{\text {control }}-\mathrm{Abs}_{\text {extract }}\right) / \mathrm{Abs}_{\text {control }}\right] \times 100
$$

where $\mathrm{Abs}_{\text {control }}$ represents the absorbance of the control and $\mathrm{Abs}_{\text {extract }}$ denotes the absorbance of the experimental sample.

\subsubsection{Determination of cytotoxicity}

The NIH/3T3 (ATCC@ CRL-1658 ${ }^{\mathrm{TM}}$, mouse embryonic fibroblast), a normal/healthy cell line, was used to assess the in vitro cytotoxicity of GTPS. Cell viability was evaluated by using the [2,3-bis-(2-methoxy-4-nitro-5-sulphophenyl)-2H-tetrazolium5-carboxanilide] (XTT) cytotoxicity test kit (Xenometrix AG, Allschwil, Switzerland), as outlined by Altintop et. al. (2012) and Kwiecińska et. al. (2012). Cells were grown in Dulbecco's Modified Eagle Medium (DMEM) containing fetal bovine serum and penicillin-streptomycin at $37^{\circ} \mathrm{C}$ in a $5 \% \mathrm{CO}_{2}$ incubator. $\mathrm{Cy}$ totoxicity studies were carried out for nine different GTPS concentration (ranged from 5 to $0.019 \mathrm{mg} / \mathrm{mL}$ ) prepared with sterile water. Growth and solvent controls were run in four replicates. The number of cells added to the wells was $1 \times 10^{4}$ and the incubation time of the GTPS was for 24 hours. At the end of the incubation period, the upper part of the wells was removed and $200 \mu \mathrm{L}$ of fresh medium was added. After that, $50 \mu \mathrm{L}$ of XTT II and XTT I mixture (1:100) were added and held for 3 hours incubation. The absorbance values were read at 480 and $690 \mathrm{~nm}$ and $\%$ inhibition values were calculated for each concentration of GTPS. The inhibitor concentration $50\left(\mathrm{IC}_{50}\right)$ values of the GTPS were calculated. The $\mathrm{IC}_{50}$ values were estimated by plotting a dose response curve of the inhibition (\%) versus test compound concentrations.

$$
\begin{gathered}
\text { Inhibition }(\%)=100-\left[\left(\mathrm{OD}_{\mathrm{GTPS}}-\mathrm{OD}_{\text {blank }}\right) \times 100\right) / \\
\left.\left(\mathrm{OD}_{\text {solvent control }} \times \mathrm{OD}_{\text {blank }}\right)\right]
\end{gathered}
$$

where OD $_{\text {GTPS }}$ represent the optical density of GTPS, OD blank optical density of blank, and $\mathrm{OD}_{\text {solvent control }}$ optical density of solvent control.

\subsubsection{Determination of genotoxicity}

AMES microplate format (MPF) 98/100 mutagenicity assay sample kit (Xenometrix AG, Allschwil, Switzerland) was used to evaluate the genotoxicity of the selected compounds as previously described by Altintop et al. (2012, 2016). Salmonella typhimurium TA98 (frameshift mutations) and TA100 (base-pair substitutions) strains were used. The compounds were tested at the concentrations between 16 and $5,000 \mu \mathrm{g} / \mathrm{mL}(5.0,2.5,1.25$, $0.625,0.3125$, and $0.156 \mathrm{mg} / \mathrm{mL}$ in dimethyl sulphoxide in accordance with the guidelines (Chandrasekaran et al., 2011). Mutagenity was tested with/without metabolic activation with Aroclor ${ }^{\mathrm{TM}}-1254$ induced male Sprague-Dawley rat liver microsomal enzyme (S9) mix (Xenometrix AG, Allschwil, Switzerland). Growth medium (GM) was added to vials containing Salmonella typhimurium TA98 and TA100 strains. Bacterial suspension and GM were added to the culture tube. Mixture was incubated for $12-16$ hours at $37^{\circ} \mathrm{C}$ while stirring at $250 \mathrm{rpm}$. If the $\mathrm{OD}_{600}$ value was greater than 2.5 , the experiment procedure was continued. Dilutions of $10 \mu \mathrm{L}$ were prepared at 6 different concentrations (5, $2.5,1.25,0.625,0.3125$, and $0.156 \mathrm{mg} / \mathrm{mL}$ ) of GTPS. Exposures with TA98 and TA100 bacterial strains with 2-aminoanthracene, which were positive controls in the presence of the S9 enzyme fraction, and 4-nitro-o-quinoline and 2-nitrofluren, in the absence of the S9 enzyme fraction, were performed. Exposure medium, bacterial strains, GTPS, and S9 enzyme fraction were added in exposure plate and incubated for $90 \mathrm{~min}$. At the end of the period, a purple indicator medium was added to each wells and the whole contents were transferred to a 384-plate and incubated at $37{ }^{\circ} \mathrm{C}$ for 48 hours. The bacterial metabolism on the plate changed the $\mathrm{pH}$ and the purple colour turned to yellow. The average number of positive (yellow) wells based on the applied doses was calculated from triplicate replicates. The results were compared with the baseline (baseline $=$ mean number of positive wells of the zerodose control \pm 1 standard deviation) and the fold changes for each dose was determined.

Fold induction over the solvent control and fold induction over the baseline were calculated as follows: Fold induction over the solvent control was the ratio of the mean number of positive wells for the dose concentration divided by the mean number of positive wells for the zero-dose control. Fold induction over the baseline was the ratio of the mean number of positive wells for the dose concentration divided by zero-dose baseline. If the baseline was less than 1 , the value was set to 1 for calculation. All doses were compared according to student's t-test at $P<0.05$ for statistical significance.

Assessment criteria of cultures have been determined based on high and low growth rates (Flückiger-Isler and Kamber, 2012). The same test design was repeated by adding the S9 liver microsomal enzyme fraction (Xenometrix AG, Allschwil, Switzerland) obtained from rat liver, to identify the mutagenesis of GTPS in the case of metabolic activator presence. Mutagenicity of compounds 


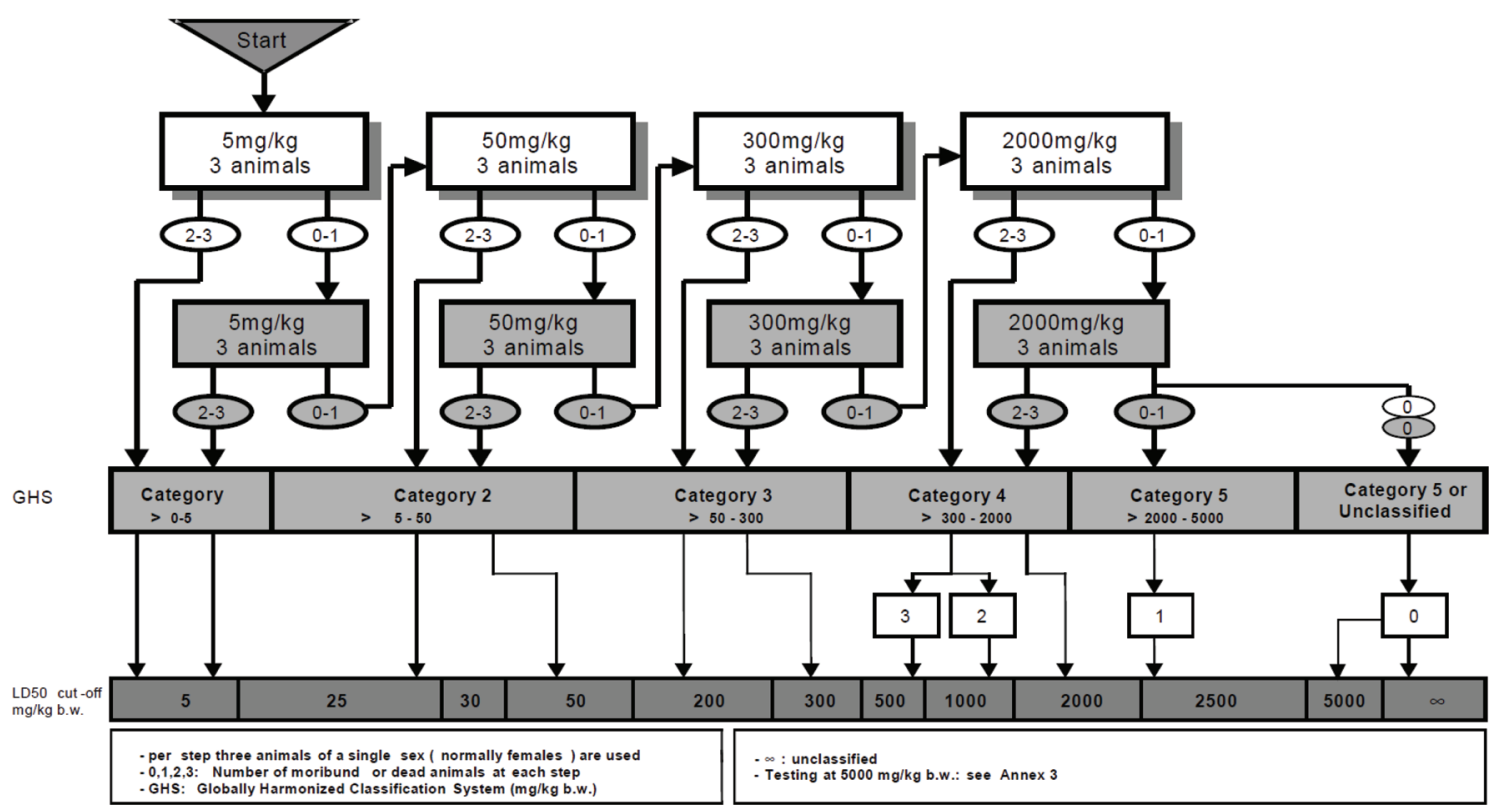

Figure 1. Limit test procedure.

was identified according to the evaluation criteria given in Table 1.

\subsubsection{Determination of lethal dose $50\left(\mathrm{LD}_{50}\right)$}

The $\mathrm{LD}_{50}$ assay was performed for GTPS according to the proto- col "OECD 423-Acute Oral Toxicity (acute toxicity classification method)" which was applied as a conventional acute toxicity test. The $\mathrm{LD}_{50}$ value of the GTPS was determined by applying the limit test protocol as specified in Annex 3 of the OECD 423 test protocol (Figure 1). The method was carried out as described by Jiang and Zhen (2004). According to the test procedure, the 8-12 weeks old

Table 2. Yields, compositional characteristics, and antioxidant activities of green tea polysaccharides

\begin{tabular}{llc}
\hline Parameters & Units & GTPS \\
\hline Yield & $\%$ & $3.05 \pm 0.15$ \\
Total carbohydrates & $\%$ & $34.33 \pm 2.00$ \\
Protein & $\%$ & $2.21 \pm 0.05$ \\
Uronic acid & $\%$ & $15.11 \pm 1.55$ \\
Total phenolics & $\mathrm{g} \mathrm{GAE} / 100 \mathrm{~g} \mathrm{GTPS}$ & $5.80 \pm 0.52$ \\
ABTS & $\mathrm{mg} \mathrm{TE} / 100 \mathrm{mg} \mathrm{GTPS}$ & $42.51 \pm 1.03$ \\
FRAP & $\mathrm{mg} \mathrm{FeSO} \cdot 7 \mathrm{H}_{2} \mathrm{O} / 100 \mathrm{mg}$ of GTPS & $19.86 \pm 1.52$ \\
Monosaccharide composition & & $3.97 \pm 0.00$ \\
Mannose & $\mathrm{mol} \mathrm{ratio} \%$ & $5.95 \pm 0.00$ \\
Ribose & & $3.06 \pm 0.00$ \\
Rhamnose & & $2.02 \pm 0.26$ \\
Glucuronic & & $0.95 \pm 0.23$ \\
Galacturonic & & $16.61 \pm 0.00$ \\
Glucose & & $40.22 \pm 0.02$ \\
Galactose & & $1.37 \pm 0.02$ \\
Xylose & & $24.13 \pm 0.01$ \\
Arabinose & & $1.72 \pm 0.03$ \\
Fucose & & \\
\hline
\end{tabular}

Abbreviations: ABTS, 2,2'-azino-bis (3-ethylbenzothiazoline-6-sulphonic acid); FRAP, ferric reducing antioxidant potential; GAE, gallic acid equivalents; GTPS, green tea polysaccharides; $T E$, trolox equivalents. Data are expressed as the mean $\pm \mathrm{SD}(n=3)$. 


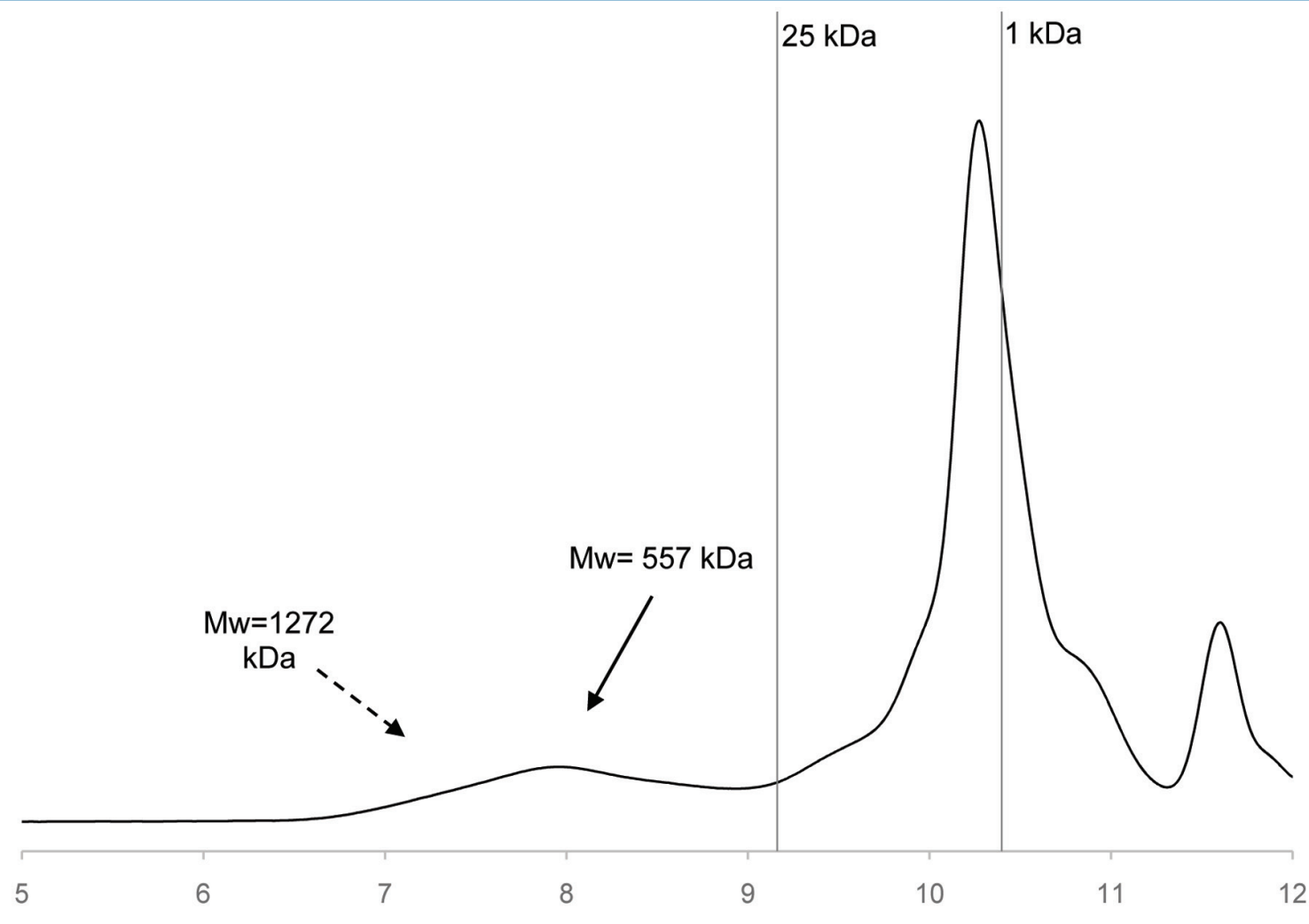

Figure 2. Molecular weight distribution of green tea polysaccharides.

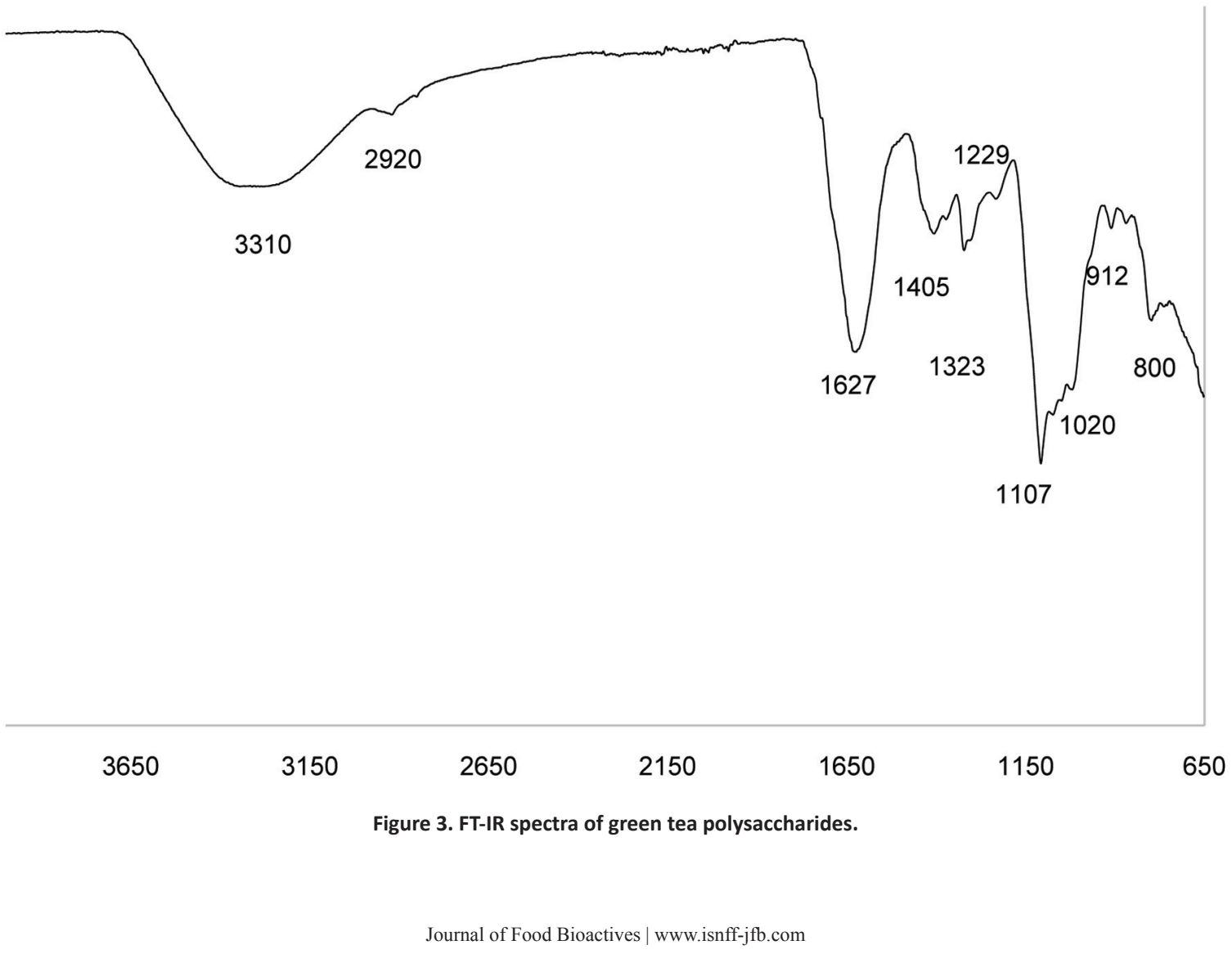


Table 3. Inhibitory effects of green tea polysaccharides on $\alpha$-glycosidase activity

\begin{tabular}{ll}
\hline Concentration $(\mathrm{mg} / \mathrm{mL})$ & Inhibition (\%) \\
\hline 2.5 & $95.25 \pm 3.13$ \\
1.25 & $93.41 \pm 3.30$ \\
0.5 & $91.86 \pm 0.04$ \\
0.125 & $43.77 \pm 1.73$ \\
0.025 & $7.15 \pm 5.25$ \\
\hline
\end{tabular}

Data are expressed as the mean $\pm \operatorname{SD}(n=3)$.

3 CD1 female mice housed in the animal facility of Genetic Engineering and Biotechnology Institute of TÜBİTAK MAM. For in vivo study, ethics was approved by the animal ethics committee of the TÜBİTAK MAM (Gebze-Kocaeli, Turkey).

\subsection{Statistical analysis}

Differences were considered to be significant at $P \leq 0.05$. Differences for analyses were estimated by analysis of variance (ANOVA). Statistical analyses were performed using the SPSS 18.0 version (SPSS Inc., Chicago, IL).

\section{Results and discussion}

\subsection{Compositional characteristics and antioxidant activities of GTPS}

The yield, compositions, and antioxidant activities of GTPS are given in Table 2. The yield of crude polysaccharides was 3.05\%, similar to those found by Cai et al. (2013) and Chen et al. (2009).
The uronic acid and neutral sugar content of GTPS were 15.11 and $34.33 \%$, respectively. GTPS was a protein bounded polysaccharide and, therefore, its protein content was $2.21 \%$. The monosaccharide composition of GTPS was mainly composed of galactose, arabinose, and glucose with the presence of some glucuronic and galacturonic acids. The latter two were acidic heteropolysaccharide. Our results are similar to the studies in the literature showing that tea polysaccharides have arabinogalactan structures (Chen et al., 2009; Scoparo et al., 2016).

Total phenolic content and antioxidant activities, using two different assays, were also determined (Table 2). Total phenolic content of GTPS was $5.80 \mathrm{~g} \mathrm{GAE} / 100 \mathrm{~g}$ sample. Wang et al. (2013) revealed that tea polyphenols are the major antioxidant in the crude tea polysaccharide and when only polysaccharide fractions isolated from crude polysaccharide extract, these fractions showed low antioxidant activity. Similar to their observation, GTPS in the present study showed low antioxidant activities when evaluated by FRAP and ABTS assays (Table 2).

\subsection{Molecular weight distribution}

The molecular weight distributions and peak pattern of GTPS are

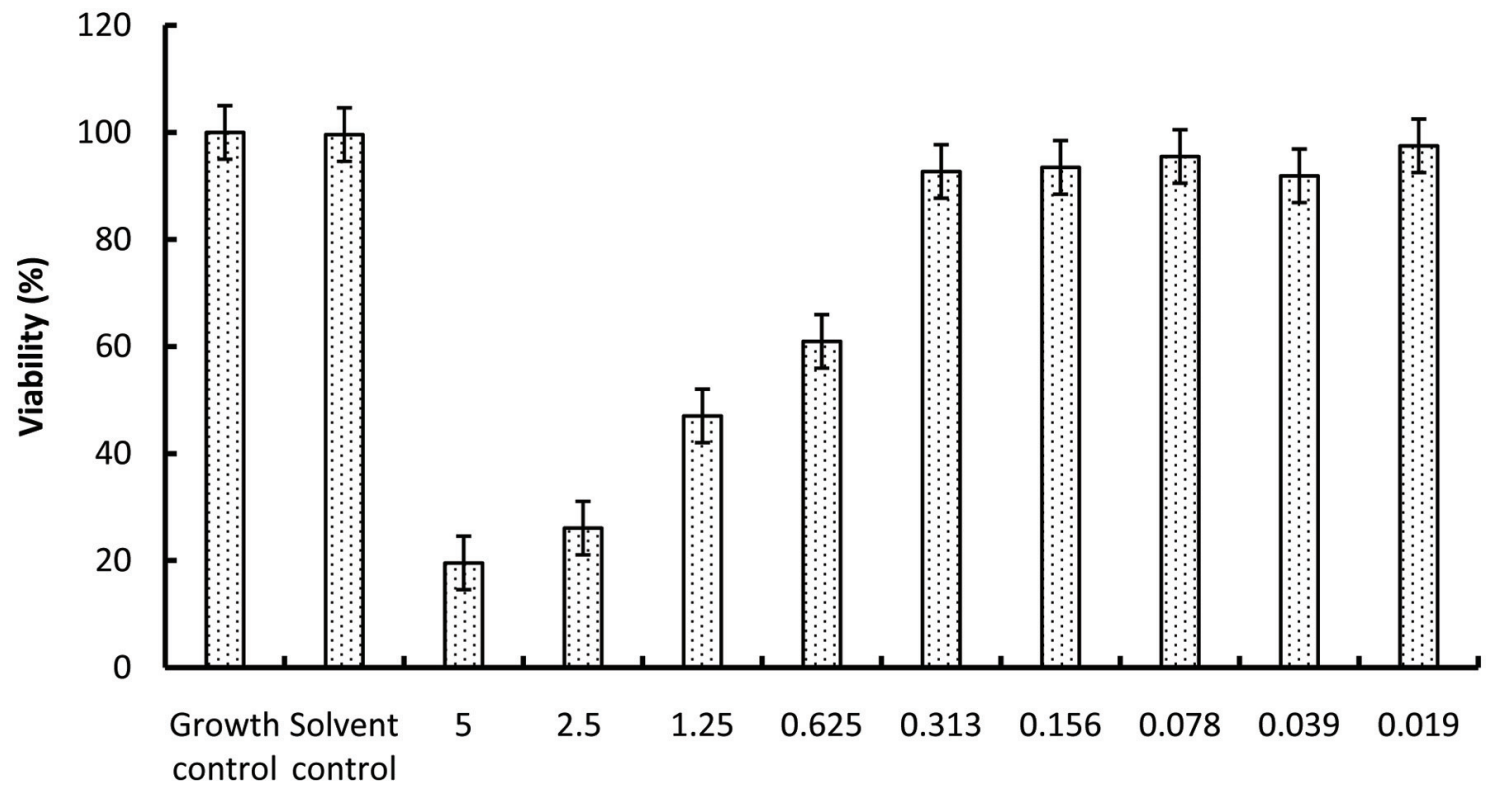

\section{Concentration $(\mathrm{mg} / \mathrm{mL})$}

Figure 4. Percentage viability of green tea polysaccharides. Data are expressed as the mean \pm SD $(n=3)$. 


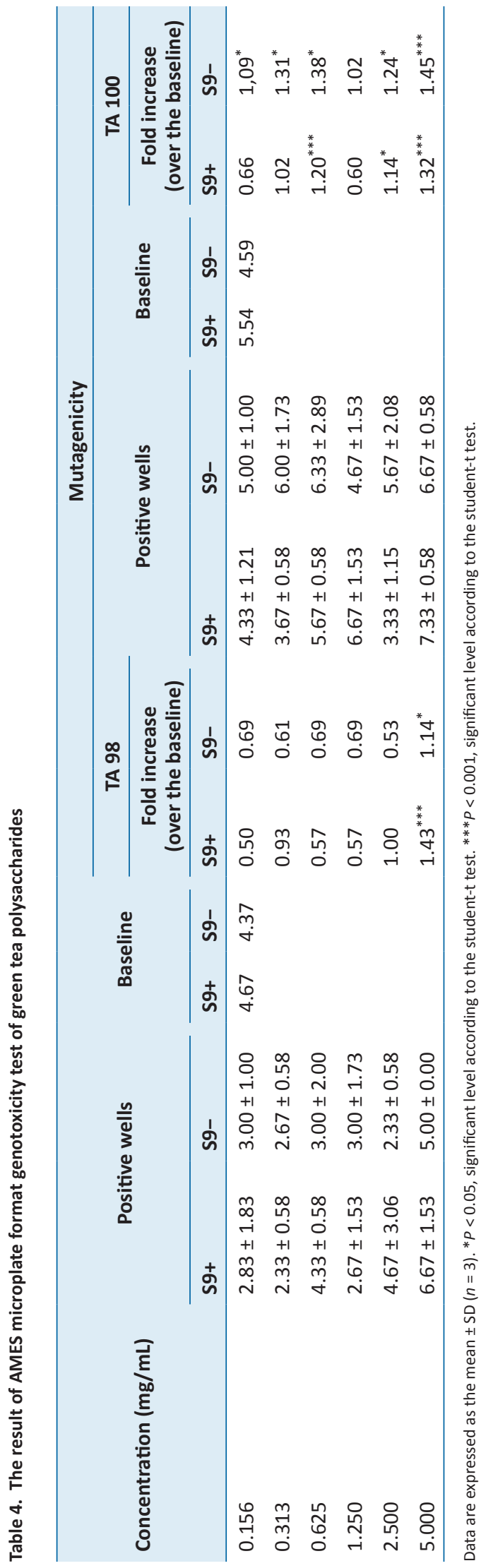

illustrated in Figure 2. Weight-average molecular weight (Mw) value, calculated by the regression equation $\left[\log (\mathrm{Mw})=-0.081 \mathrm{x}^{3}\right.$ $+2.215 \mathrm{x}^{2}-20.965 \mathrm{x}+72.956 ; R^{2}=0.998$, were obtained by dextran standards and retention time]. GTPS contained three peaks and predominant component $(81.48 \%)$ was eluted at a retention time of $10.27 \mathrm{~min}$ with a $\mathrm{Mw}$ value of $2.07 \mathrm{kDa}$. The minor fractions eluted at a retention time of $7.89 \mathrm{~min}(9.96 \%)$ and 11.60 $(8.55 \%)$ had $\mathrm{Mw}$ of 1,409 and $9 \mathrm{kDa}$, respectively. Chen et al. (2016) reviewed that the $\mathrm{Mw}$ of tea polysaccharides ranged from 1.2 to $3,900 \mathrm{kDa}$ and explained that the differences may have resulted from raw material, extraction, as well as separation and determination methods.

\subsection{FT-IR analysis of GTPS}

The FT-IR spectra of GTPS showed characteristic peaks for polysaccharides (Figure 3). Peaks at 1,000-1,200, 2,800-3,200, and $3,200-3,600 \mathrm{~cm}^{-1}$ are characteristic for polysaccharides, the broadly intense peak at $3,310 \mathrm{~cm}^{-1}$ represented the stretching vibrations of $\mathrm{O}-\mathrm{H}$, while the weak band at $2,920 \mathrm{~cm}^{-1}$ was attributed to the $\mathrm{C}-\mathrm{H}$ stretching and bending vibrations. The relatively strong absorption peak at $1,627 \mathrm{~cm}^{-1}$ was in association with absorbed water. A combination of two intensive bands at 1,405 and 1,323 $\mathrm{cm}^{-1}$ were characteristic of $\mathrm{C}-\mathrm{O}$ stretch and $\mathrm{C}-\mathrm{H}$ or $\mathrm{OH}$ bending. The broad band at 1,107 and $1,020 \mathrm{~cm}^{-1}$ suggested the presence of $\mathrm{C}-\mathrm{O}-\mathrm{H}$ side groups and $\mathrm{C}-\mathrm{O}-\mathrm{C}$ glycosidic band vibration and should be the characteristic absorption bands of pyran glycosides. The peaks around 910 and $800 \mathrm{~cm}^{-1}$ corresponded to skeletal modes of pyranose rings, which was characteristic of $\alpha$-type glycosidic linkages between the sugar units (Chen et al., 2009).

\subsection{Inhibitory effects on $\alpha$-glucosidase activity}

As shown in Table 3, GTPS had a dose-dependent effect on $\alpha$-glucosidase inhibitory activity and the inhibition rose from 7.15 to $91.86 \%$ as the concentration increased from 0.025 to $0.5 \mathrm{mg} /$ $\mathrm{mL}$. Increasing the concentration further did not alter the inhibition ratio. As a positive control, at $1.25 \mathrm{mg} / \mathrm{mL}$ of acarbose concentration, $27.80 \%$ inhibition on $\alpha$-glucosidase was observed whereas GTPS showed $93.41 \%$ inhibition.

The $\alpha$-glucosidase inhibitors are of current interest owing to their promising therapeutic potential for diabetic treatment as oral hypoglycaemic agents. The $\alpha$-glucosidase inhibitors act on the brush border of intestinal mucosa to inhibit the post-meal blood glucose level from rising and decrease fasting blood glucose, to some extent, by delaying the carbohydrates digestion and absorbance at intestine (Wei et al., 2010).

\subsection{Toxicity tests of GTPS}

GTPS were evaluated for their toxicity by performing cytotoxicity and genotoxicity tests. Additionally, the $\mathrm{LD}_{50}$ value of GTPS was determined to assess the safe use level as a dietary supplement.

According to the results of cytotoxicity test, $\mathrm{IC}_{50}$ value was determined as $0.98 \mathrm{mg} / \mathrm{mL}$. This result shows that the GTPS was not cytotoxic at the active concentrations (Figure 4). Below 0.625 $\mathrm{mg} / \mathrm{mL}$ concentrations, GTPS reached viability values similar to growth control which indicates the safety of it. In addition, AMES MPF assay was used to identify the genotoxicity of GTPS (Table 4). More than 25 positive wells were observed with positive and negative controls. The results showed less than 8 positive wells 
TA $9859(+)$

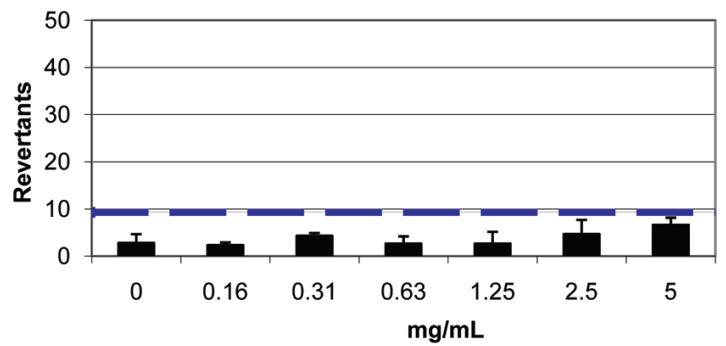

TA 100 s9(-)

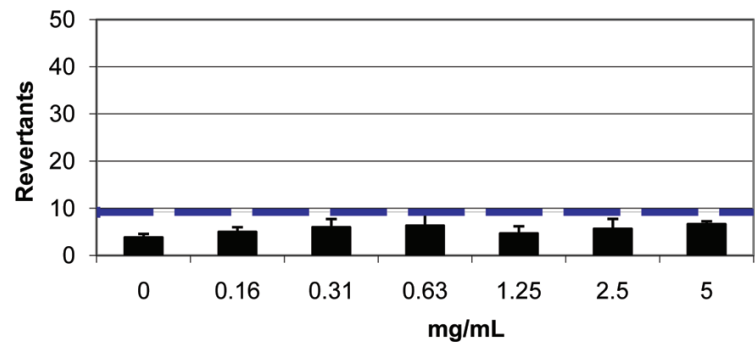

TA 98 S9 (-)

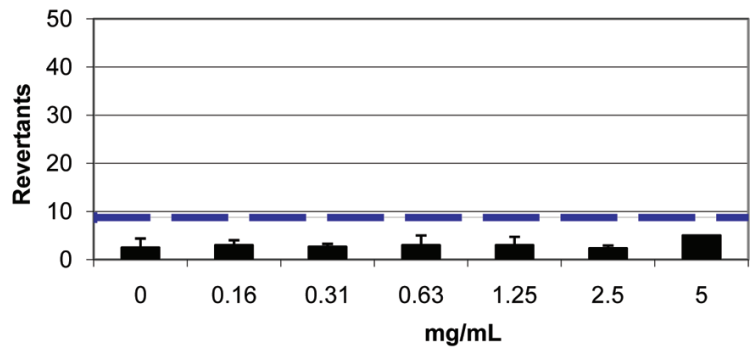

TA $100 S 9$ (+)

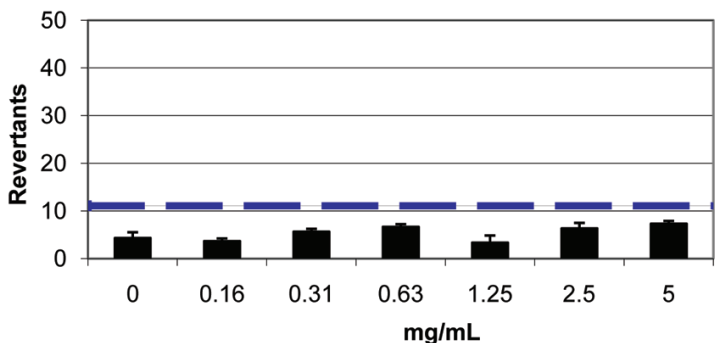

Figure 5. The results of AMES microplate format test of green tea polysaccharides in TA 98 and TA 100 strains (- 2-fold induction over the baseline).

in the presence and absence of S9 with TA98 and TA100; which are the requirements of AMES MPF test validation. It was also observed that GTPS did not reach any of the indicated increases at baseline in the presence and absence of the S9 enzyme fraction in both strains (Figure 5). Furthermore, although GTPS has significant differences at some doses, no significant dose-response tendency was observed between the consecutive doses $(P>0.05)$. Therefore, GTPS had no mutagenic effect according to AMES test results.

The limit test protocol was applied to identify the $\mathrm{LD}_{50}$ value of GTPS as specified in Annex 3 of the OECD 423 test protocol. GTPS was administrated to mice by gavage with $5,000 \mathrm{mg} / \mathrm{kg}$ dose level. Animals were monitored for mortality and after 5 days the experiment was terminated. None of the GTPS administrated mice died during the trial. Following single dose gavage application of the sample, the experimental animals were observed at short intervals for the first 4 hours. Abnormal clinical findings were also absent in animals during the entire experiment. Significant weight loss was not observed in the test mice $(>10 \%)$ and normal liver weight index values were recorded. At the end of the observation period, all mice were subjected to gross pathology examination. As expected, no pathological findings were noticed in the examinations. According to these results and the survival of all three mice, GTPS was categorized as "GHS 5 or unclassified" according to the OECD 423-Acute Oral Toxicity (acute toxicity classification method) test method.

\section{Conclusion}

GTPS was a protein bounded acidic polysaccharide and had arabinogalactan structure. $\alpha$-Glucosidase inhibitory activity of GTPS was dose dependent and it was $93.41 \%$ at a concentration of 1.25 $\mathrm{mg} / \mathrm{mL}$, whereas acarbose, as synthetic anti-diabetic drug, only showed $27.80 \%$ inhibition at the same concentration. Due to its potential to be considered as an antidiabetic dietary supplement in food and medicinal fields, GTPS were further evaluated for toxicity which demonstrated no cytotoxic and no mutagenic effects. The acute toxicity study also showed that the $\mathrm{LD}_{50}$ of GTPS was greater than $5,000 \mathrm{mg} / \mathrm{kg}$ and it was categorized as "HHS 5 or unclassified". All these findings suggest that GTPS can be employed as an antidiabetic dietary supplement without posing any potential health risk.

\section{Acknowledgments}

This study is a part of project funded by TÜBİTAK (under 1003 programme-project no 1050037). We are grateful to ÇAYKUR Tea Enterprise for providing tea samples.

\section{References}

Alasalvar, C., Pelvan, E., Özdemir, K.S., Kocadağlı, T., Mogol, B.A., Paslı, A., Özcan, N., Özçelik, B., and Gökmen, V. (2013). Compositional, nutritional, and functional characteristics of instant teas produced from low- and high-quality black teas. J. Agric. Food Chem. 61: 7529-7536.

Altintop, M.D., Ozdemir, A., Turan-Zitouni, G., Ilgin, S., Atli, O., Iscan, G., and Kaplancikli, Z.A. (2012). Synthesis and biological evaluation of some hydrazone derivatives as new anticandidal and anticancer agents. Eur. J. Med. Chem. 58: 299-307.

Altintop, M.D., Atli, O., Ilgin, S., Demirel, R., Ozdemir, A., and Kaplancikli, Z.A. (2016). Synthesis and biological evaluation of new naphthalene substituted thiosemicarbazone derivatives as potent antifungal and anticancer agents. Eur. J. Med. Chem. 27: 406-414.

Blumenkrantz, N., and Asboe-Hansen, G. (1973). New method for quantitative determination of uronic acids. Anal. Biochem. 54: 484-489.

Bradford, M.M. (1976). A rapid and sensitive method for the quantitation 
of microgram quantities of protein utilizing the principle of proteindye binding. Anal. Biochem. 72: 248-254.

Cai, W., Xie, L., Chen, Y., and Zhang, H. (2013). Purification, characterization and anticoagulant activity of the polysaccharides from green tea. Carbohyd. Polym. 92: 1086-1090.

Chandrasekaran, C.V., Sundarajan, K., Gupta, A., Srikanth, H.S., Edwin, J., and Agarwal, A. (2011). Evaluation of the genotoxic potential of standardized extract of Glycyrrhiza glabra (GutGard ${ }^{\mathrm{TM}}$ ). Regul. Toxicol. Pharmacol. 61: 373-380.

Chen, H., Qu, Z., Fu, L., Dong, P., and Zhang, X. (2009). Physicochemical properties and antioxidant capacity of 3 polysaccharides from green tea, oolong tea, and black tea. J. Food Sci. 74: 469-474.

Chen, G., Yuan, Q., Saeeduddin, M., Ou, S., Zeng, X., and Ye, H. (2016). Recent advances in tea polysaccharides: Extraction, purification, physicochemical characterization and bioactivities. Carbohyd. Polym. 153: 663-678.

Cheng, H., Feng, S., Jia, X., Li, Q., Zhou, Y., and Ding, C. (2013). Structural characterization and antioxidant activities of polysaccharides extracted from Epimedium acuminatum. Carbohyd. Polym. 92: 63-68.

Dai, J., Wu, Y., Chen, S., Zhu, S., Yin, H., Wang, M., and Tang, J. (2010). Sugar compositional determination of polysaccharides from Dunaliella salina by modified RP-HPLC method of precolumn derivatization with 1-phenyl-3-methyl-5-pyrazolone. Carbohyd. Polym. 82: 629-635.

DuBois, M., Gilles, K.A., Hamilton, J.K., Rebers, P.A., and Smith, F. (1956). Colorimetric method for determination of sugars and related substances. Anal. Chem. 28: 350-356.

Flückiger-Isler, S., and Kamber, M. (2012). Direct comparison of the Ames microplate format (MPF) test in liquid medium with the standard Ames pre-incubation assay on agar plates by use of equivocal to weakly positive test compounds. Mutat. Res. 747: 36-39.

Horszwald, A., and Andlauer, W. (2011). Characterisation of bioactive compounds in berry juices by traditional photometric and modern micro- plate methods. J. Berry Res. 1: 189-199.

Jiang, H.Y., and Zhen, G.L. (2004). Studies of tea polysaccharides on lowering blood sugar of mice. Food Sci. 25: 166-169.

Kwiecińska, P., Taubøll, E., and Gregoraszczuk, E.t. (2012). Effects of valproic acid and levetiracetam on viability and cell cycle regulatory genes expression in the OVCAR-3 cell line. Pharmacol. Rep. 64: 157-165.

Nie, S.P., and Xie, M.Y. (2011). A review on the isolation and structure of tea polysaccharides and their bioactivities. Food Hydrocolloid. 25: 144-149.

Scoparo, C.T., Souza, L.M., Dartora, N., Sassaki, G.L., Santana-Filho, A.P., Werner, M.F.P., Borato, D.G., Baggio, C.H., and lacomini, M. (2016). Chemical characterization of heteropolysaccharides from green and black teas (Camellia sinensis) and their anti-ulcer effect. Int. J. Biol. Macromol. 86: 772-781.

Shori, A.B. (2015). Screening of antidiabetic and antioxidant activities of medicinal plants. J. Integr. Med. 13: 297-305.

OECD. (2001). Test Guideline 423. Acute Oral Toxicity - Acute Toxic Class Method. OECD Publishing, Paris, France.

Xi, X., Wei, X., Wang, Y., Chu, Q., and Xiao, J. (2010). Determination of tea polysaccharides in Camellia sinensis by a modified phenol-sulfuric acid method. Arch. Biol. Sci. 62: 669-676.

Xiao, J.B., and Jiang, H. (2015). A review on the structure-function relationship aspect of polysaccharides from tea materials. Crit. Rev. Food Sci. 55: 930-938.

Wang, S., and Zhu, F. (2016). Antidiabetic dietary materials and animal models. Food Res. Inter. 85: 315-331.

Wang, Y., Zhao, Y., Andrae-marobela, K., Okatch, H., and Xiao, J. (2013). Tea polysaccharides as food antioxidants: An old woman's tale? Food Chem. 138: 1923-1927.

Wei, X., Chen, M., Xiao, J., Liu, Y., Yu, L., Zhang, H., and Wang, Y. (2010). Composition and bioactivity of tea flower polysaccharides obtained by different methods. Carbohyd. Polym. 79: 418-422. 